Research Article

\title{
Validation of Unsteady Thermodynamic CFD Simulations of Aircraft Wing Anti- Icing Operation
}

\author{
B. Balakrishna ${ }^{\dot{\mathrm{A}^{*}}}$ and V Gangadhar Praveen Ketha ${ }^{\dot{\mathrm{A}}}$ \\ ${ }^{\dot{A}}$ Mechanical Engineering Department, Jawaharlal Nehru Technological University Kakinada, Andhra Pradesh, India \\ Accepted 10 January 2014, Available online 01 February 2014, Special Issue-2, (February 2014)
}

\begin{abstract}
In-flight icing is a major concern in aircraft safety and a non-negligible source of incidents and accidents, and is still a serious hazard today. It remains consequently a design and certification challenge for aircraft manufacturers. The aerodynamic performance of an aircraft can indeed degrade rapidly when flying in icing conditions, leading to incidents or accidents. In-flight icing occurs when an aircraft passes through clouds containing super cooled water droplets at or below freezing temperature. Droplets impinge on its exposed surfaces and freeze, causing roughness and shape changes that increase drag, decrease lift and reduce the stall angle of attack, eventually inducing flow separation and stall. This hazardous ice accretion is prevented by the use of dedicated anti-icing systems, among which hot-air-types are the most common for turbofan aircraft. A three-dimensional unsteady thermodynamic simulation model is used to describe the dynamic response of an aircraft wing anti-icing system. This computational fluid dynamics based model involves a complete wing segment including thermal anti-icing bay inside the leading edge. The unsteady, integrated internal/external thermal flow simulation is presented with heat conductivity through the solid skin in a fine mesh. The calculated skin temperature results are satisfactory in their good match with flight test data. The presented research work indicates a strong potential of using computational fluid dynamics in dynamic wing anti-icing system model development and validation.
\end{abstract}

Keywords: Anti-Icing, Unsteady Simulations, Thermal Analysis, Aircraft wing anti-icing system (WICS).

\section{Introduction}

\subsection{In-Flight Icing}

It is understood that, In-flight icing is a major concern in aircraft safety, a non-negligible source of accidents and is still a serious hazard today. As a consequence, it remains a design and certification challenge for the aircraft manufacturers.

Indeed, the aerodynamic performance of an unprotected aircraft flying in icing conditions can degrade rapidly and if not treated appropriately, lead to incidents and accidents. In-flight icing generally occurs at or below the freezing point when an aircraft passes through clouds containing super cooled droplets.

Beside the risks of decreasing pilots' visibility and putting in jeopardy the efficiency of the aircraft radar, communication antennas and probes, in-flight icing can considerably affect the aerodynamic as well as control and stability performances of the aircraft.

Droplets impinge on the exposed surfaces of the aircraft and freeze, increasing surface roughness and inducing early boundary-layer transition to turbulent flow. Ice accretion also leads rapidly to increased drag, decreased lift, with a corresponding increase in stall speed

*Corresponding author: B. Balakrishna

DOI: http://dx.doi.org/10.14741/ijcet/spl.2.2014.88 and decrease in stall angle which constitute propitious conditions for flow separation and stall even at sometimes significantly lower angles of attack, especially in the maneuver, holding, take-off and landing phases. It will also modify the pressure distribution and the load dispatch, induce vibrations and decrease the aircraft's maneuverability. In-flight icing is also an issue for engines and propellers, degrading performances, blocking inlets, and possibly damaging in case of ice ingestion. Fig.1(Mathieu Paul Constantin Pellissier, 2010 ) shows in detail.

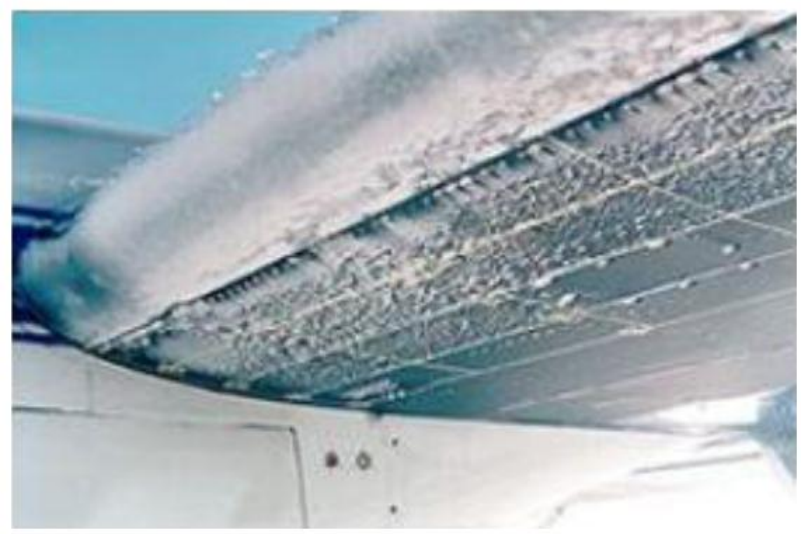

Fig.1 Ice formation on aircraft wing section.

475 | International Conference on Advances in Mechanical Sciences 2014 


\subsection{In-Flight Icing Protection: Anti-Icing/De-Icing}

To avoid such events, aircraft are equipped with systems to prevent ice accretion on the exposed critical aerodynamic and control surfaces during flight. These anti-icing systems must comply with flight safety regulations outlined by national certification authorities such as the FAA (Federal Aviation Administration), the EASA (European Aviation Safety Agency) and Transport Canada, or other governmental entities.

As opposed to ground icing, which can be visually checked and taken care of on the runway, in-flight icing requires rigorous procedures and systems to address flight safety regulations outlined by national certification authorities. Such systems include ice detection systems coupled to ice protection systems (de-icing or anti-icing systems), usually located at the leading edge of the exposed surfaces. De-icing systems are reactive and commonly consist in mechanically deformable membranes or electro-impulse devices. Such systems are used periodically to remove already accreted ice. Anti-icing systems, such as hot-bleed-air circulation systems or electro-thermal devices, are preemptive and designed to prevent ice accretion by evaporating the impinging droplets.

One of the most widely used anti-icing devices for wings, stabilizer and engine nacelles of commercial and corporate turbofan engine aircraft is a high-temperature bleed-air anti-icing system, commonly called Piccolo tube. This system circulates hot-air, collected from the engine's first compressor, to the areas to be protected.

Fig.2 (Mathieu Paul Constantin Pellissier, 2012 ) shows in detail.

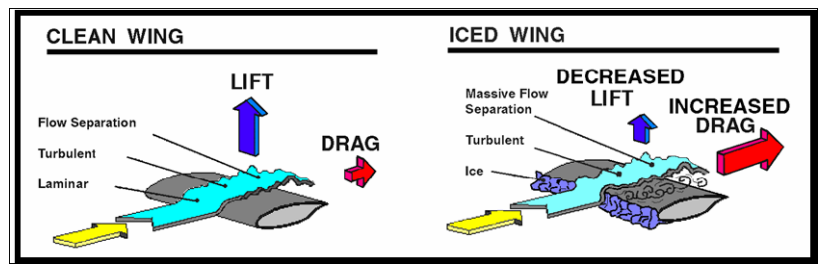

Fig.2 Aerodynamic effects of in-flight icing

\subsection{Objective of the Current Work}

The research background comes from the necessity of developing a dynamic control technique for aircraft wing anti-icing system (WAIS). Fig.3 shows a typical flowchart of such a system. The hot air from the turbo engine is introduced to the leading edge through pipes and valves. The control module regulates the wing anti-icing valve (WAIV) to control the heat flux into the Piccolo tubes in the leading edge anti-icing bays. It in turn adjusts the skin temperature of the wing leading edge. The control system works in a closed loop with the temperature sensors located at several points of the wing leading edge surface.

An efficient wing anti-icing control system development requires a dynamic thermal fluid model. It is a challenging task due to its complicated physics behavior in both anti- and de-icing flight operations. One wellreceived approach is to develop an empirical dynamic model of parameters that are tuned by flight test data, which are expensive and difficult to obtain, and even impossible in the early design phase. Instead, our research explores using the computational fluid dynamics (CFD) simulation data to assist in dynamic model development. Obviously unsteady simulations are required to serve this purpose. Fig.3 ( Jun Hua, Fanmei Kong, Hugh H. T. Liu) explains the procedure in detail as shown below.

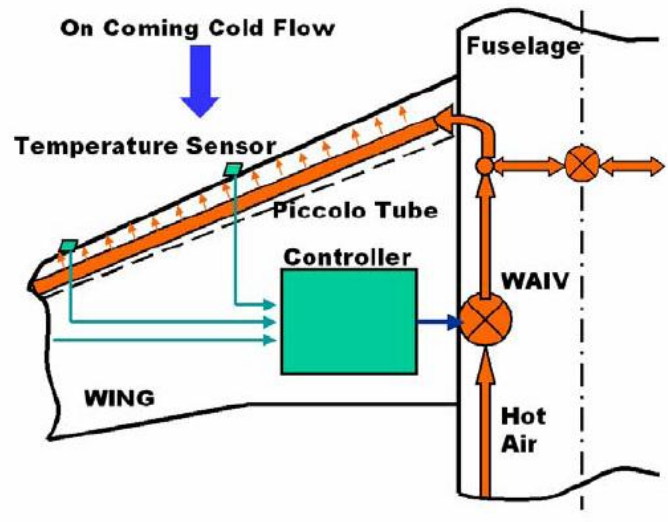

Fig.3 Flowchart of a WAIS (left hand side only)

CFD has been used in anti-icing research for years. Topics of recent work are mainly covered in the application of available CFD tool Ansys-Fluent for aircraft anti-icing device design.

In this paper, the 3D unsteady CFD simulations are first studied for time-dependent computations of WAIS under different hot air inlet conditions. They simulate the thermal flow under the adjustment of the control valves, and calculate the corresponding skin temperature. The calculated skin temperature results are found to coincide with the flight measurement very well. Therefore, the unsteady simulation results may be used in dynamic model tuning to complement or even re-create a flight test process.

\section{Model Desperation}

The 3D wing span segment (Fig. $4 \& 5$ ) consists of the following components. 1) A Piccolo tube introduces the hot air into the leading edge anti-icing bay. There are a number of small hoses on the front side of the Piccolo tube from where the hot jets impinge the inner surface of the leading edge. The small holes are located staggered in three rows, one on the central chord plane, other two of angles with $15^{\circ}$ upper and lower from the wing chord plane. 2) The aluminium skin is heated by the hot air in the anti-icing operation. 3) Two exhaust holes on the lower side of the bay allow the hot air to exit to the external flow. 4) Two ribs separate the bays in the span direction with the hoses on the ribs neglected. 5) The heat shield serves as the back wall of the bay.

Tetrahedral meshes are generated for both internal and external flow fields. The interior and exterior meshes are connected through the mesh in the exhaust holes. The same tetrahedral mesh is also used inside the aluminium skin for the heat conductivity calculation. The higher parallel computational power made it possible to conduct 
the 3D unsteady simulation on a common PC, which has an Intel Core i5 processor, 4G memory.

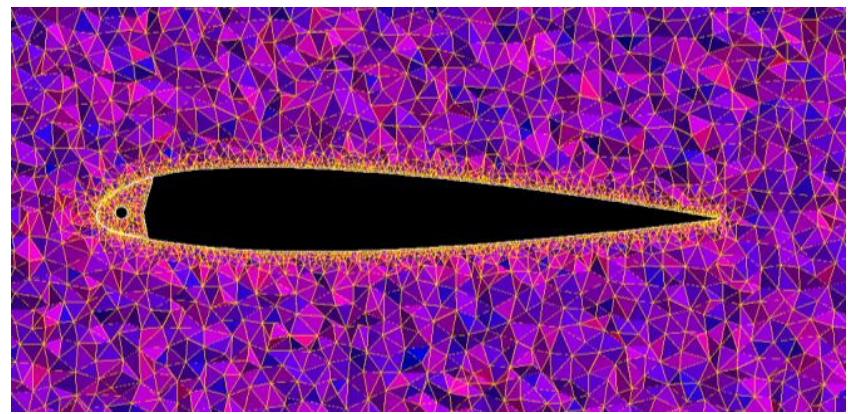

Fig.4 Mesh generation on wing segment

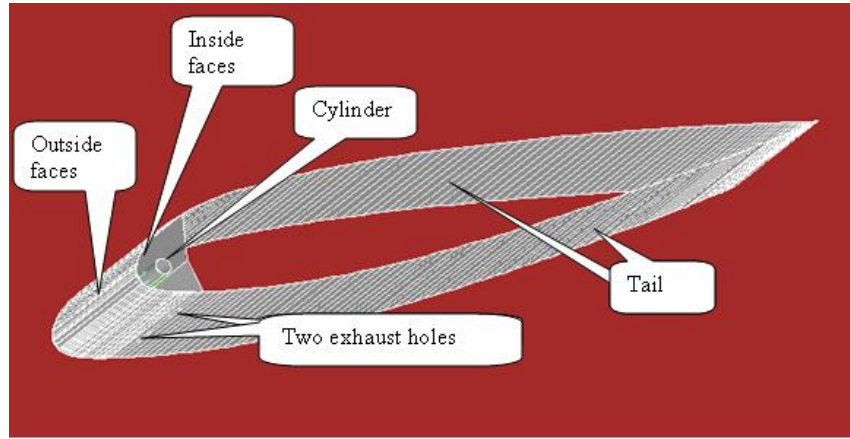

Fig.5 3D view of wing segment

\section{Grid/Mesh Independence Test}

Grid convergence is the term used to describe the improvement of results by using successively smaller cell sizes for the calculations. A calculation should approach the correct answer as the mesh becomes finer, hence the term grid convergence.

The normal CFD technique is to start with a coarse mesh and gradually refine it until the changes observed in the results are smaller than a pre-defined acceptable error.

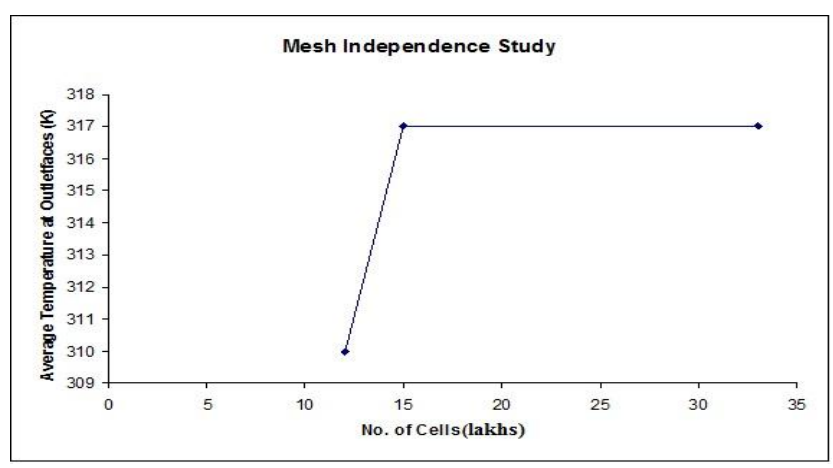

Fig.6 Mesh Independence Study graph

\section{Unsteady Thermodynamic Simulations}

\subsection{The Navier-Stokes Solver}

The CFD method used in this research is a well-known Navier-Stokes solver FLUENT. Its reliability has been demonstrated by a great number of aerospace and industrial applications.

\subsection{The Boundary Conditions for the Unsteady Simulation}

For the integrated internal/external thermal flow simulation, pressure far field boundary condition (B.C.) is used for the external field, which includes the flight Mach number, angle of attack and altitude. Pressure inlet B.C. is given at the Piccolo tube holes, where the total pressure and total temperature change with the regulation of the wing anti-icing valve. Symmetrical B.C.'s are applied on the side faces of the external flow field and the sidewalls of the solid skin. Heat transfer is considered for the inner and outer surfaces of the leading edge skin. Other wing surfaces are treated as adiabatic walls.

On the one hand, most of the anti-icing operations are taking place in the second phase climb and initial phase of cruise, and the far field conditions may not vary sharply. On the other hand, the Piccolo tube pressure and temperature will change quickly with the adjustment of the control valves. In the CFD analysis, the Piccolo tube inlet boundary conditions are controlled by using the userdefined functions (UDF). In this approach, the inlet pressure and temperature are described by functions of time written in $\mathrm{C}^{++}$language. In each time step of the flow solution, the B.C. values will be calculated and assigned to the solver automatically.

4.3 Unsteady Simulation with Basic Functions as the Piccolo Tube Inlet Conditions

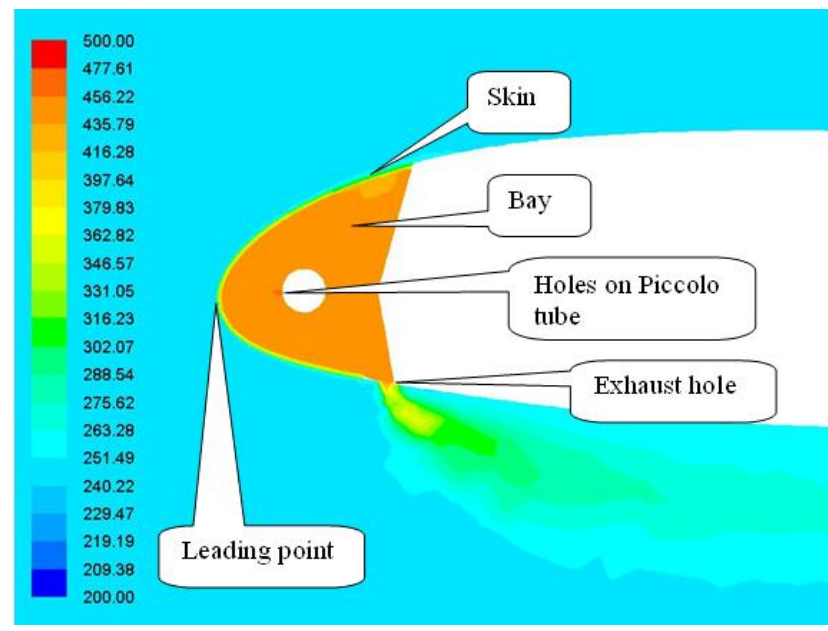

Fig.7 Temperature contour of the $3 \mathrm{D}$ model at $\mathrm{t}=100 \mathrm{sec}$

Most control systems require dynamic input and output variables. In the case of a WAIS, such variables include the pressure and temperature of the piccolo tube, as functions of time. In order to simulate the dynamic functions, one will use the step or exponential functions for approximation. At the initial step of the unsteady investigation, three such functions are selected to describe the Piccolo tube inlet heat fluxes in a 3D model:

1) Single step : $F(X, t)=X_{t=0}+D X, t>0$

477 | International Conference on Advances in Mechanical Sciences 2014 
2) Exponent law: $F(X, t)=X_{t=0}+D X(1-\exp (-k t))$, $\mathrm{k}=0.1$ and 0.5

3) Sin law: $F(X, t)=X_{t=0}+D X * \operatorname{Sin}(2 \pi k t), \mathrm{k}=120$

Where $X=T_{0}$ and $P_{0} ; T_{0} t=o=453 \mathrm{~K}$,

$P_{0 t=o}=90000 \mathrm{~Pa} ; D T_{o}=5 \mathrm{~K}, D P_{O}=2500 \mathrm{~Pa}$

for all the above cases. Meanwhile, the external flow condition is for flight at $\mathrm{M}=0.31, \alpha=4.5^{\circ}$ and altitude $6500 \mathrm{~m}$.

Figure 6 shows clearly the hot temperature in the bay, clod external field, skin temperature variation and the merge of the hot exhaust with the external flow.

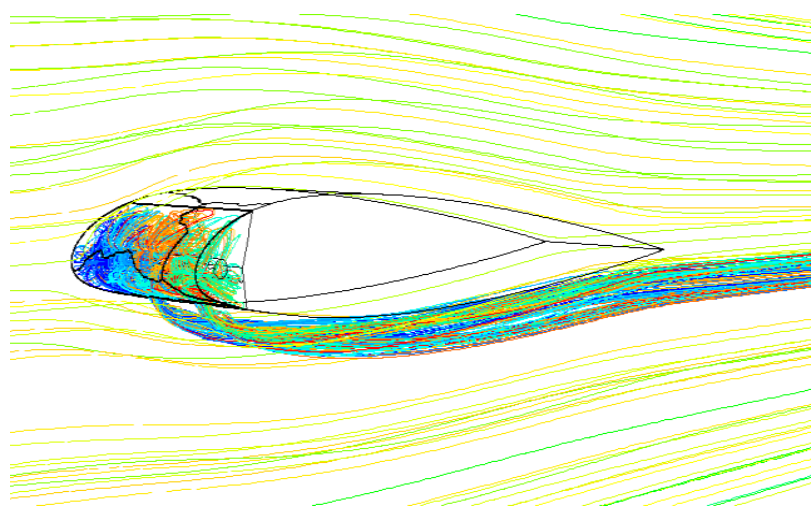

Fig.8 Streamlines of the CFD simulation colored by path lines

Figure 9 shows how the temperature of the different surfaces changing with the Piccolo tube inlet conditions for the first two functions.



Fig.9 Temperature response at different surfaces

\section{Results}

We are simulating actual flight wing conditions using CFD. Hot air temperature coming from piccolo tube is $453 \mathrm{~K}$ and ambient temperature $\sim 250 \mathrm{~K}$ convection phenomenon has been observed, this hot air coming from the holes in piccolo tube is used to heat the airfoil skin surface and we can observe the airfoil skin temperature increasing with time. These following figures show clearly the hot temperature in the bay, cold external field, skin temperature variation and the merge of the hot exhaust with the external flow.

5.1 Skin temperature variation history of the CFD simulation $(K)(t=10,60,90,100$ seconds $)$

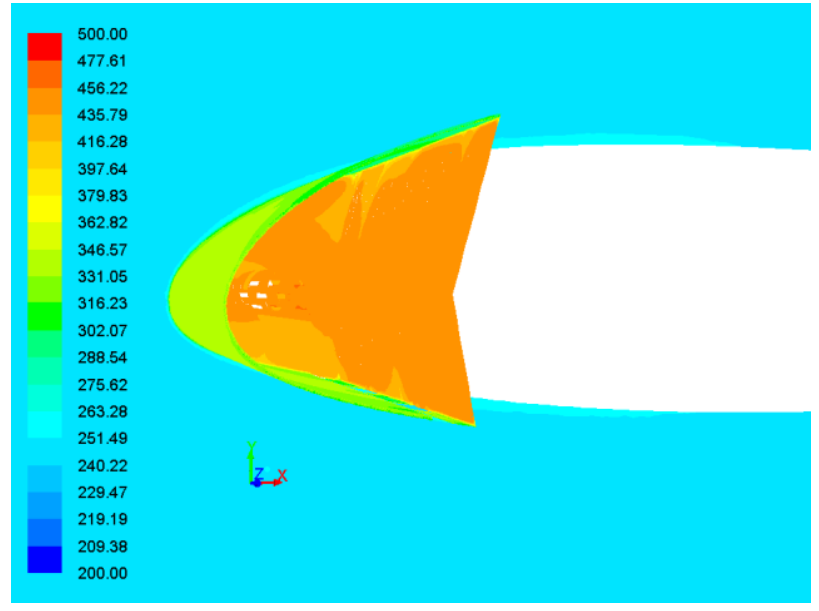

Fig.10 Skin temperature variation for $10 \mathrm{sec}$

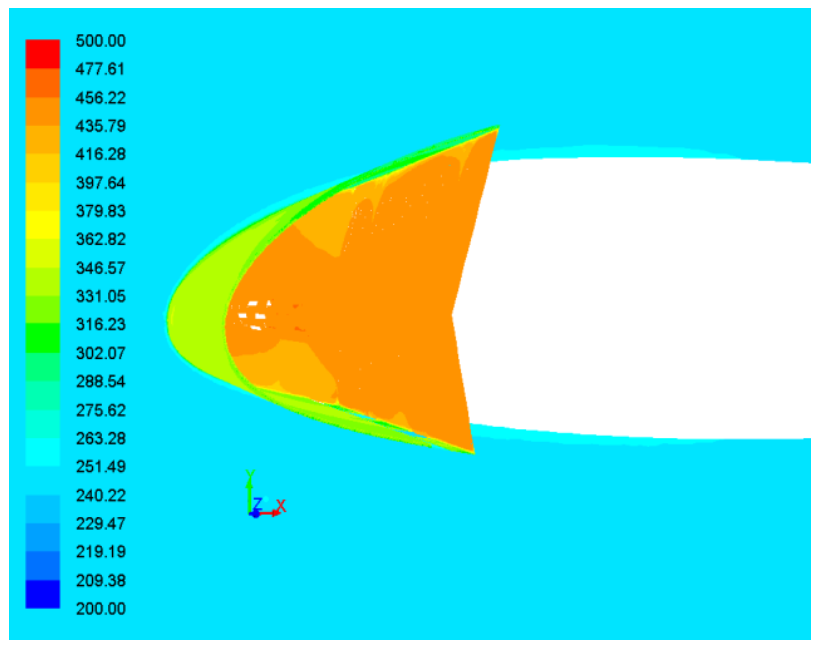

Fig.11 Skin temperature variation for $60 \mathrm{sec}$

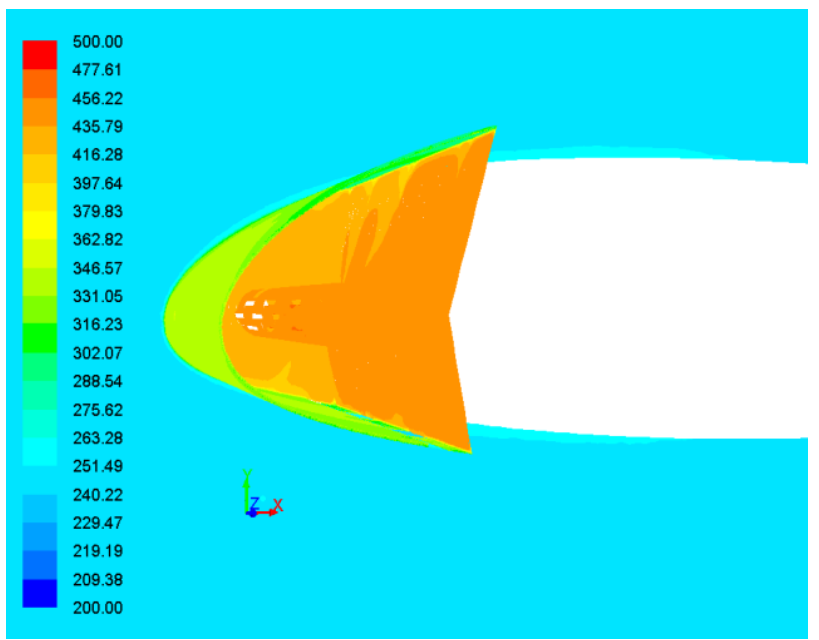

Fig.12 Skin temperature variation for $90 \mathrm{sec}$

478 | International Conference on Advances in Mechanical Sciences 2014 


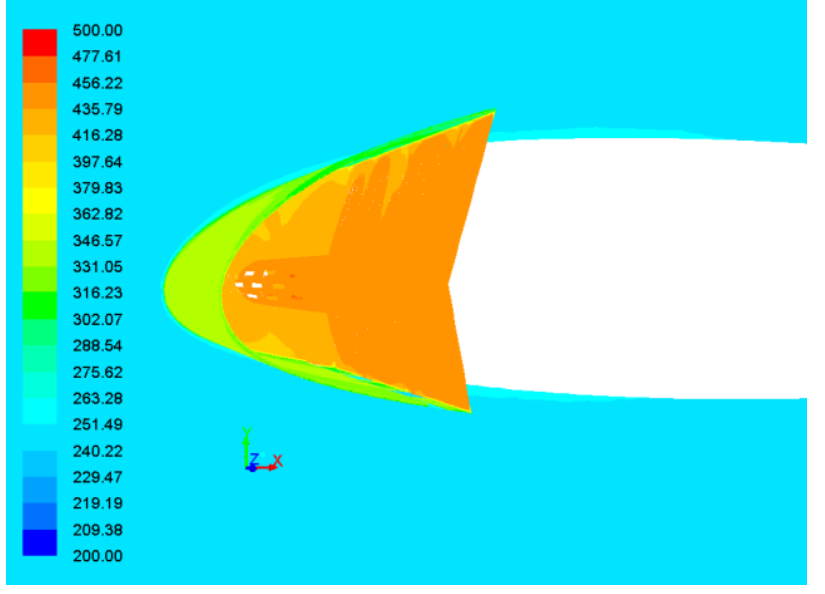

Fig.13 Skin temperature variation for $100 \mathrm{sec}$

\section{Verification \& Validation}

To verify our CFD results we have used three functions as described in inlet functions section and found, the CFD results doesn't vary much

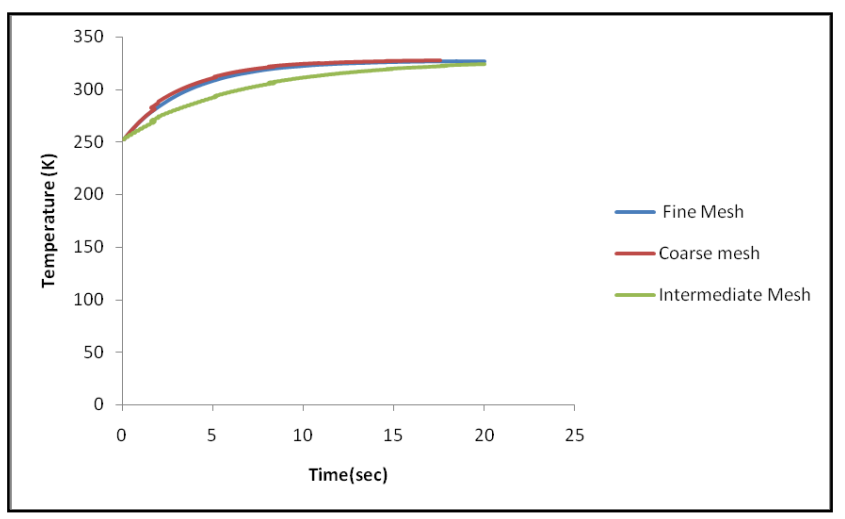

Fig.14 Temperature responses at 3 conditions

\subsection{Comparison with Flight Test}

It could be seen that the $3 \mathrm{D}$ CFD results have excellent coincidence with the flight test, in the entire time segments from the WAIV opening and regulating period.

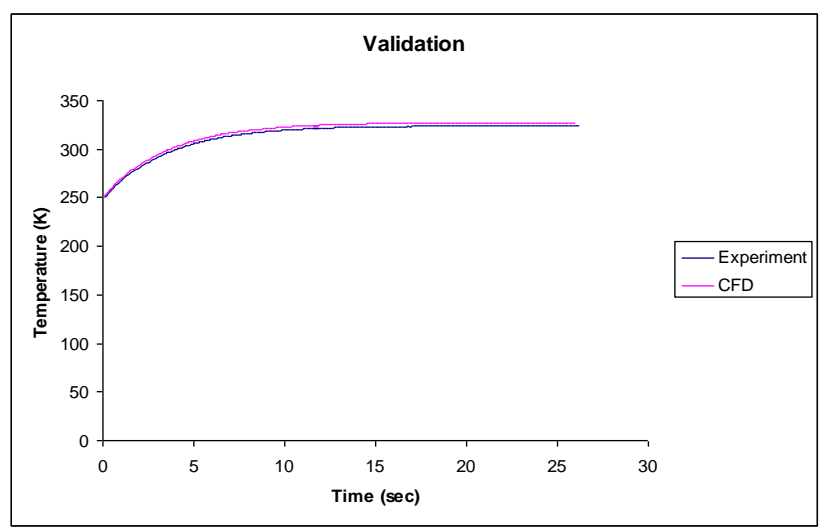

\section{Conclusions}

An aircraft wing anti-icing flight test loop has been represented with a 3D unsteady thermodynamic CFD simulation, based on a Navier-Stokes method.

The heating process is simulated at the beginning of this study by applying different basic functions presenting Piccolo tube heat flux, and the wing skin responses are discussed.

The 3D CFD model involves a complete wing segment with the piccolo type thermal anti-icing bay. In the unsteady integrated internal/external thermal flow simulation with heat conductivity through the solid skin, time dependent boundary condition specifications and proper time steps are investigated. The tetrahedral mesh generated increases the unsteady simulations efficiency.

The calculated 3D skin temperature dynamic variation coincides with the flight measurements very well. It indicates the possibility of applying CFD simulation data to the anti-icing system development. It may be used in dynamic model tuning, to complement or even be used in place of the flight test data that are expensive and often not complete enough to serve this purpose. The process of integrating such unsteady CFD simulations into WAIS dynamic model development is currently under investigation.

\section{Acknowledgement}

The authors would like to thank Akula Chaturvedi, the Asst Surveyor at Indian Register of Shipping (Research Division) for his valuable inputs during the different phases of the project.

\section{References}

Thesis submitted by MATHIEU PAUL CONSTANTIN PELLISSIER of Computational Fluid Dynamics Laboratory, Department of Mechanical Engineering, McGill University, Montréal, Québec, Canada in June 2010.

Jun Hua, Fanmei Kong, Hugh H. T. Liu. AJER Article title Unsteady Thermodynamic CFD Simulations of Aircraft Wing Anti-Icing Operation. American Journal of Engineering Top International Journal

Bourgault, Y., Boutanios, Z. and Habashi, W.G., Three-dimensional Eulerian Approach to Droplet Impingement Simulation Using FENSAP-ICE, Part1: Model, Algorithm, and Validation, Journal of Aircraft, Vol. 37, No. 1, pp 95-103, 2000.

Beaugendre, H., Morency, F. and Habashi, W.G., FENSAP-ICE's ThreeDimensional In-Flight Ice Accretion Module: ICE3D, Journal of Aircraft, Vol.40, No.2, pp 239-247, 2003.

Bidwell, C.S., Pinella, D. and Garrison, P., Ice Accretion Calculations for a Commercial Transport Using the LEWICE3D, ICEGRID3D and CMARC Programs, NASA/TM-1999-208895, 1999.

Morency, F., Tezok, F. and Paraschivoiu, I., Heat and Mass Transfer in the Case of Anti-Icing System Simulation, Journal of Aircraft, Vol. 37, No.2, pp245-252, 2000.

de Mattos, B.S. and Oliveira, G.L., Three-dimensional Thermal Coupled Analysis of a Wing Slice Slat with a Piccolo Tube, AIAA-2000-3921, 2000.

Liu, H.H.T. and Hua, J., Three-Dimensional Integrated Thermodynamic Simulation for Wing Anti-Icing System, Journal of Aircraft, Vol. 41, No. 6, pp 1291-1297, 2004

Hua, J. and Liu, H.H.T., Fluid Flow and Thermodynamic Analysis of a Wing Anti-Icing System, Canadian Aeronautics and Space Journal, Vol. 51, No. 1, pp 35-40, 2005.

Fluent 14 User Guide, 2013.

Bombardier Aerospace, Flight Test Data of Wing Anti-Icing System, Internal Communication, 2003

Fig.15 Temperature plots at Outer surface for Experimental vs. CFD results 